\title{
TRAZABILIDAD BOVINA EN EL SUDOESTE BONAERENSE: REFLEXIONES DESDE EL ENFOQUE NEOINSTITUCIONAL ${ }^{1}$ CATTLE TRACEABILITY IN THE SOUTHWEST OF BUENOS AIRES: REFLECTIONS FROM THE NEO-INSTITUTIONALISM APPROACH
}

Mauricia Nori², Liliana Scoponi ${ }^{3}$, Regina Durán 4 , Marcelo Fernandes Pacheco Dias 5

Fecha de recepción: 11/08/2015 Fecha de aceptación: 25/11/2015

\section{Resumen}

La trazabilidad se ha difundido en diferentes países para garantizar una oferta de productos con buenas condiciones higiénico-sanitarias y nutricionales, empleándose en sistemas de certificación de calidad y gestión de las cadenas agroalimentarias. En la Argentina, los avances con este alcance son aún insuficientes en la cadena de la carne bovina. Se analizan, así, las presiones coercitivas, normativas y cognitivas sobre trazabilidad y su influencia en la gestión de la ganadería del Sudoeste bonaerense, actividad clave para el desarrollo local y nacional, mediante una investigación cualitativa a partir de documentos y entrevistas semiestructuradas. Se concluye que la Argentina ha avanzado en regulaciones sobre identificación animal para control sanitario y fiscal, sin una implementación holística y con visión comercial del sistema. Varios factores inciden para que las presiones normativas y cognitivas sean débiles en pos del aseguramiento de la calidad, 
como políticas de exportación, complejidad de las disposiciones y asimetrías del mercado interno.

Palabras clave: trazabilidad, cadena bovina, calidad, presiones institucionales, neoinstitucionalismo.

\section{Abstract}

In the present context, traceability has spread in different countries to ensure a range of products with good sanitary and nutritional conditions. Although in Argentina the topic has been discussed, there has been very little progress in the cattle chain. Cognitive, regulatory and coercive pressures and their influence on the cattle management in the Southwest Buenos Aires are analyzed through a semi-qualitative research from documents and semi structured interviews. Cattle raising is crucial for both local and national development. We conclude that Argentina has made progress in animal identification for health and fiscal control but without a holistic implementation and lacking a commercial view of the system. Several factors influence to weaken the regulatory and cognitive pressures that ensure quality: export policies, complex regulations and asymmetry in the internal market.

Keywords: traceability, bovine chain, quality, institutional pressures, neo-institutionalism.

JEL: M11; Q130. 


\section{Introducción}

En el contexto actual, el sector agroalimentario debe satisfacer demandas de consumidores cada vez más exigentes respecto de la calidad e inocuidad de los alimentos. Para dar respuesta a ello, la trazabilidad se ha difundido en diferentes países como mecanismo de información para garantizar una oferta de productos con buenas condiciones higiénico-sanitarias y nutricionales. Según el Codex Alimentarius, la trazabilidad es la capacidad de seguir el movimiento de un alimento a través de etapa(s) especificada(s) de la producción, transformación y distribución. La norma ISO 8402 define la trazabilidad como la posibilidad de reencontrar los antecedentes, la localización de una entidad, mediante identificaciones registradas. Por lo tanto, la trazabilidad debe permitir identificar cualquier producto agroalimentario en cualquier actividad de la cadena de valor: interna de la empresa y externa del agronegocio. Esta posibilidad de rastreo a lo largo del proceso productivo conlleva varios beneficios: control del proceso, corrección de errores de manera oportuna, planificación para actuar en forma preventiva, confiabilidad de la cadena productiva y del producto mismo, trabajo conjunto de los diferentes eslabones de la producción y comercialización, información al consumidor sobre quién y cómo obtuvo el producto para garantizar la sanidad alimentaria y lograr ventajas competitivas de diferenciación (Felipe Boente \& Briz Escribano, 2004).

Los orígenes de la trazabilidad se encuentran en las crisis sanitarias sufridas por los países desarrollados en las cadenas de carnes hacia fines de la década del 80 y durante la década del 90. En 1986 aparece por primera vez en una explotación bovina del Reino Unido, la encefalopatía espongiforme bovina (EEB) conocida como "mal de la vaca loca", que brota como epidemia en la Unión Europea (UE) en los años 1996 y 2000. Asimismo, en diciembre de 2003, siete estados de Estados Unidos estaban en riesgo y treinta países que representaban el $90 \%$ de las exportaciones de ese país habían prohibido el ingreso de ganado, carne y sus derivados, generando pérdidas de mercado significativas. A esta difícil situación se le sumó una importante crisis de fiebre aftosa a partir de febrero de $2001 \mathrm{y}$ otras crisis sanitarias de carnes sustitutas como la peste porcina clásica en Bélgica, los 
pollos contaminados con dioxinas en 1999 y, más recientemente, la gripe aviar en 2004. Si bien la fiebre aftosa y la peste porcina no son transmisibles al hombre, causaron grandes pérdidas económicas en Reino Unido y otros países de Europa Continental (Aráoz, 2004; Green, 2007; Rodríguez-Ramírez, González-Córdova, Arana, Sánchez- Escalante \& Vallejo-Córdoba, 2010).

Durante el 2003 apareció en Estados Unidos una cepa tóxica de Escherichia coli que produce el síndrome urémico hemolítico en el ser humano. En la Argentina se han dado varios casos por consumo de hamburguesas. También se ha encontrado contaminación de alimentos con listerias, salmonella y otros patógenos. Finalmente, los productos de uso veterinario están siendo pasibles de mayor control, de modo que cumplan su función terapéutica sin afectar al consumidor con residuos (Aráoz, 2004). Green (2007) destaca que si bien en la actualidad no se habla más de "crisis de la vaca loca", hay dos elementos importantes a tener en cuenta. El primero es que aún se siguen detectando algunos casos aislados en Europa y el segundo es que la trazabilidad, a la que dio lugar esta problemática sanitaria en la carne bovina, luego fue generalizada al conjunto de los alimentos a partir de las experiencias citadas.

En el último decenio, las exportaciones mundiales de carne vacuna aumentaron un $20 \%$ y evidenciaron grandes cambios, principalmente por el crecimiento de Brasil, que ha superado a la UE y EE. UU., regiones que se recuperan lentamente luego de la irrupción del "mal de la vaca loca" (De las Carreras, 2010). En cambio, la Argentina ha quedado relegada al onceavo lugar, con ventas cercanas a $187.000 \mathrm{t}$ anuales de res con hueso, siendo sus principales destinos Rusia y Alemania (IPCVA, 2012). No obstante, el escenario internacional es favorable y brinda oportunidades de crecimiento. Emergen importantes desafíos para la ganadería argentina; en particular, la trazabilidad surge como necesidad, dado que en un futuro próximo no será posible colocar productos alimenticios que no hayan sido certificados de acuerdo a normas de aseguramiento de la calidad. Si bien en el país se viene discutiendo sobre el tema desde hace años, aún no se ha avanzado lo suficiente en la materia (Aráoz, 2004).

Dentro del contexto presentado, la vertiente sociológica del neoinstitucionalismo en la teoría de la organización puede contribuir a una 
mejor comprensión de las presiones del entorno que afectan a los actores de la cadena bovina y aportar explicaciones a los problemas de coordinación y modernización. Carvalho \& Vieira (2003) destacan que las transformaciones en el mundo moderno requieren tener en cuenta dimensiones socioculturales, además de orientaciones racionalistas de eficiencia, puesto que ha ido arraigándose a través del tiempo la noción de que la actividad humana está muy integrada a contextos institucionales, donde los valores, las normas y la cultura tienen relevancia (Meyer, 2008). Así, las presiones ejercidas por las regulaciones formales (leyes y disposiciones administrativas), los estándares normativos acordados por organismos profesionales o bien las creencias y las percepciones sobre cómo llevar a cabo la actividad agraria están aumentando en los agronegocios para satisfacer los requisitos cada vez más exigentes de calidad, producción sustentable y ética de los bienes de origen agroindustrial, pudiendo incluso presentarse de manera conflictiva o contradictoria (Pache \& Santos, 2010).

El neoinstitucionalismo sociológico no ha sido demasiado explorado en el campo de las actividades de origen agropecuario; sin embargo, se advierte una preocupación reciente (Machado-da-Silva \& Coser, 2006; Heyder \& Theuvsen, 2009; Lopes \& Baldi, 2013), ya que dicho enfoque contempla las reglas y los elementos sociales a los que las organizaciones deben ajustarse para lograr legitimidad y apoyo social. Por lo tanto, se considera de interés introducir reflexiones bajo esta óptica que resulten complementarias de otros abordajes, como la nueva economía institucional, ampliamente difundida para el estudio de los agronegocios, en especial a través de las investigaciones de la escuela PENSA (Programa de Estudos dos Negócios do Sistema Agroindustrial) de la Universidad de São Paulo.

El presente trabajo, el cual representa un avance de una investigación más amplia, tiene por objetivo describir las demandas contextuales coercitivas, normativas y miméticas sobre trazabilidad que inciden en la cadena ganadera bovina argentina, e identificar la influencia que ejercen para su gestión en el Sudoeste (SO) bonaerense. En particular, se procura profundizar el análisis de las regulaciones coercitivas, dada la profusión de disposiciones emanadas por diferentes organismos en los últimos años en la Argentina, que generan interrogantes sobre su efectividad en la gestión de la 
actividad ganadera y el posible condicionamiento de las prácticas sociales sobre protocolos de origen y calidad, derivadas de las restantes presiones. Se pretende evaluar si la adopción de la trazabilidad, independientemente del doble estándar - mercado interno versus exportación-, se cumple e interpreta con carácter integral, generando condiciones para implementar estrategias de aseguramiento de la calidad.

La ganadería bovina constituye una de las cadenas agroalimentarias de mayor importancia socioeconómica a nivel nacional (De las Carreras, 2003; Iglesias \& Ghezan, 2010; Ponti, 2011). El estudio se circunscribe al SO bonaerense en virtud del impacto que presenta esta actividad para el desarrollo local, que se explica por las condiciones edafoclimáticas imperantes, las cuales limitan la diversificación productiva más allá de la ganadería vacuna y de la agricultura de cereales. Esta situación fue receptada por la Ley 13647/07 o "Ley del SO bonaerense", que crea el marco jurídico de políticas especiales para la región. Asimismo, no se han encontrado investigaciones con este alcance que reflejen las condiciones reales de utilidad del sistema de trazabilidad argentino y pudiesen apoyar dichas políticas.

El texto fue organizado con la siguiente estructura: en la primera sección, se exponen los conceptos planteados por la corriente neoinstitucional dentro de la teoría de la organización, que constituyen el fundamento teórico de la investigación; en la segunda sección, se describen sus aspectos metodológicos; en la tercera sección, se discuten los resultados hallados del análisis de las presiones coercitivas, normativas y cognitivas sobre la trazabilidad en la cadena bovina; finalmente, en la cuarta sección, se resumen las consideraciones finales.

\section{Marco teórico referencial}

La teoría institucional se ha convertido en un enfoque dominante en la macroteoría de la organización (Greenwood et al., 2008). Considera que la supervivencia y el éxito de las organizaciones dentro de la estructura competitiva de los mercados no solo dependerán de la eficiencia y el control 
de recursos clave. Será necesario, además, contemplar las presiones institucionales de sus entornos (DiMaggio \& Powell, 1983).

Estas presiones cumplen un papel importante a la hora de formar la realidad de las organizaciones y pueden ser interpretadas de manera diferente, incidiendo en su desempeño. En consecuencia, se reconoce que el ambiente institucional provee significado y estabilidad al comportamiento social, dando forma y restringiendo las acciones organizacionales (Pache \& Santos, 2010).

Para Carvalho \& Vieira (2003) esto hace posible un delineamiento más preciso de la configuración de un área de actividad, que constituye un campo organizacional, y por ende la elaboración y la implementación de políticas públicas más apropiadas para contribuir al desarrollo local. La noción de campo organizacional es la unidad de análisis del neoinstitucionalismo dentro de la teoría institucional.

Un campo organizacional comprende organizaciones que producen bienes o servicios similares, proveedores, compradores, consumidores, agencias de regulación, entre otras. Tienen en común que participan de un mismo sistema de significados e interactúan más frecuentemente entre sí que con otras (DiMaggio \& Powell, 1983).

Por lo tanto, las organizaciones que lo integran no necesariamente están ligadas por geografía u objetivos; su característica distintiva es que componen un área reconocida de vida institucional (DiMaggio \& Powell, 1983). Es así que la cadena de la carne bovina puede también analizarse bajo este concepto, dado su carácter de red de relaciones interorganizacionales, que se extiende desde la provisión de insumos a la mesa del consumidor, involucrando otros actores públicos y privados que favorecen los flujos comerciales y de información.

En un campo organizacional se encuentran presentes demandas institucionales, que son presiones de conformidad ejercidas sobre las organizaciones por los referentes institucionales (Pache \& Santos, 2010). Estas demandas pueden ser desplegadas a través de tres tipos de presiones: coercitivas, normativas y miméticas. Las presiones coercitivas se fundan en el poder regulador del Estado y suponen la existencia de un entorno legal 
común que incide sobre las organizaciones, representado por leyes, reglamentaciones, controles y sanciones (Scott, 2001). Las demandas normativas están constituidas por normas y valores que definen medios legítimos para lograr las metas deseadas. Actúan como estándares promovidos por asociaciones profesionales u otros organismos (Scott, 2001). DiMaggio \& Powell (1983) consideran que pueden difundirse a través de dos importantes canales: la profesionalización de las organizaciones, mediante la actividad de técnicos o asesores, y la participación en redes, es decir, la interacción directa con pares o bien indirecta vía asociaciones o cámaras.

Finalmente, las presiones miméticas refieren a la dimensión cognitiva de las personas para superar las limitaciones que impone la incertidumbre y encuentran su aplicación empírica en la imitación de prácticas predominantes (de uso frecuente) en un determinado campo organizacional, o que produjeron resultados positivos para otros, o bien de modelos de organizaciones percibidas como exitosas (Scott, 2001).

Si las organizaciones no se adecuan al entorno institucional, pueden sufrir, por un lado, una desventaja económica por no adaptarse a los patrones institucionales. Por otro, al no entender o compartir las mismas estructuras cognitivas que el resto de las organizaciones, podrán requerir más tiempo y esfuerzo económico y de gestión para comprender las nuevas exigencias institucionales y adaptarse a ellas. Por último, puede darse un costo social, en cuanto a la pérdida de apoyo de los stakeholders o grupos de interés clave por no contemplar sus expectativas reflejadas en las exigencias institucionales, afectando la legitimidad (Llamas Sánchez, 2005).

En el actual contexto, las organizaciones están cada vez más sujetas a demandas contradictorias impuestas por grupos de interés o stakeholders influyentes, donde satisfacer una puede implicar infringir otras. Las organizaciones que enfrentan demandas institucionales en conflicto están afectadas por múltiples y contradictorios regímenes de regulación, órdenes normativos y/o lógicas culturales (Pache \& Santos, 2010).

Esto provoca que a menudo las organizaciones se comporten de manera que desafían la lógica económica o las normas de comportamiento racional, realidad frente a la cual el neoinstitucionalismo dentro de la teoría 
institucional ofrece un paradigma dedicado a su comprensión (Suddaby, 2010).

Las investigaciones que adoptan el nivel de campo organizacional han puesto énfasis en la existencia de lógicas compitiendo dentro del mismo (Thornton \& Ocasio, 2008). A pesar de los avances significativos, aún falta un examen sistemático de la forma en que las presiones institucionales se imponen en las organizaciones y el modo en que descifran y responden a dichas demandas (Oliver, 1991). Actualmente, se reconoce que debe atenderse más a los procesos por los cuales las organizaciones comprenden y dan sentido a las presiones institucionales del entorno, considerándolas como sistemas interpretativos (Suddaby, 2010; Suddaby et al., 2010).

\section{Aspectos metodológicos}

Para cumplir los objetivos del presente estudio, el cual integra una investigación más amplia sobre la cadena bovina del SO bonaerense, se realizó un estudio descriptivo de las presiones institucionales asociadas a la trazabilidad, adoptando un enfoque cualitativo (Hernández Sampieri, Fernández Collado \& Baptista Lucio, 2010). Se utilizaron dos técnicas de recolección de datos: consulta de documentos y entrevistas semiestructuradas. La investigación en documentos fue escogida, pues permite responder a cuestiones sobre el pasado y los cambios ocurridos haciendo uso de documentos (Saunders, Lewis \& Thornhill, 2011). Se relevaron diferentes fuentes de información secundaria de organismos públicos nacionales y provinciales, leyes y normativas.

Respecto de los datos primarios recogidos a través de entrevistas, la definición de los entrevistados obedeció a los siguientes criterios: eslabón de la cadena que representan; trayectoria y conocimiento de la actividad; y grado de importancia dentro del campo organizacional. Así, las entrevistas se efectuaron a un productor de ciclo completo, a un consignatario, al gerente de un frigorífico integrado a un supermercado con alcance regional y al representante local del SENASA (Servicio Nacional de Sanidad y Calidad Agroalimentaria), organismo de control y administración del sistema de trazabilidad bovina en la Argentina. 
Las entrevistas se efectuaron en el período junio-agosto de 2014 utilizando un cuestionario en el que se agruparon los siguientes temas: trazabilidad según disposiciones vigentes como sistema integral de seguimiento para el aseguramiento de la calidad; trazabilidad como costo y/o oportunidad de lograr mejores precios; condición de aplicabilidad de los programas de calidad de carnes certificadas; y aspectos que privilegia la normativa actual en materia de identificación animal y trazabilidad. Las entrevistas fueron grabadas y transcriptas de forma de identificar los fundamentos empíricos de las cuestiones planteadas en la teoría, analizando los datos a partir de la revisión efectuada de la literatura. Para el análisis documental y de las entrevistas se empleó la técnica de análisis de contenido (Bardin, 1977).

\section{Resultados y discusión}

\subsection{Presiones coercitivas sobre trazabilidad de la carne bovina}

Un sistema de trazabilidad debe satisfacer una serie de requisitos necesarios para que sea posible su organización. En primer lugar, requiere la identificación del producto para poder efectuar su seguimiento a lo largo de la cadena de valor. En el caso de los bovinos, se realiza por animal mediante diferentes tipos de dispositivos, ya sean de tecnología simple (caravanas) o bien más compleja de carácter electrónica (chips) o genética (marcadores moleculares). En segundo lugar, debe conformarse una base de datos donde cargar la información de cada animal, la cual está generalmente informatizada. En tercer lugar, hay que incorporar la información de cada animal a la base de datos. El tipo de datos a cargar variará según el eslabón del proceso de producción y comercialización de que se trate. Es fundamental que esta información se pueda ir traspasando en sucesivas etapas de la cadena. Finalmente, los datos deben estar disponibles para terceros. Por lo tanto, un requisito fundamental para que funcione un sistema de trazabilidad es la conservación de la información registrada a lo largo del proceso, para que pueda ser transmitida a través de los diferentes actores de la cadena hasta el consumidor final (Green, 2007; Bonet de Viola, 2012). El avance en las TIC 
(tecnologías de información y comunicación) está facilitando la puesta en marcha de métodos e instrumentos que hacen factible el rastreo de los alimentos con mayor precisión, permitiendo la transmisión de datos e informes generados en los diversos controles de calidad y certificaciones realizadas en la cadena de valor (Felipe Boente \& Briz Escribano, 2004).

A continuación, se detallarán las regulaciones sobre trazabilidad e identificación animal vigentes en la Argentina, para luego exponer las experiencias de Brasil y Uruguay solo a los fines de contextualizar la situación de nuestro país en relación con los jugadores clave en la oferta mundial de carne vacuna.

\subsubsection{Situación normativa en la Argentina}

A continuación se detallan las disposiciones normativas emitidas por diferentes jurisdicciones que regulan la identificación y trazabilidad bovina en la Argentina. Del análisis de sus considerandos y contenido, se advierte que persiguen como objetivo cumplir con las exigencias impuestas por el mercado externo para garantizar la seguridad alimentaria, principalmente de la UE (tabla 1). Al mismo tiempo, procuran crear herramientas eficaces para evitar problemas sanitarios, el abigeato y la evasión fiscal. Esto último también es de interés para la hacienda con destino al mercado interno, en virtud de lo cual las normas se centran en la identificación animal y de los establecimientos de origen, pero sin implicar seguimiento. 
Tabla 1. Reglamentación de trazabilidad para la exportación

\begin{tabular}{|c|c|c|}
\hline Norma & $\begin{array}{l}\text { Organismo } \\
\text { que la } \\
\text { sancionó }\end{array}$ & Objetivo de la norma \\
\hline Res. $67 / 97$ & SENASA & $\begin{array}{l}\text { Se implementan documentos específicos para el tránsito de } \\
\text { animales con destino a faena para la UE que se acompañan con } \\
\text { un certificado sanitario }\end{array}$ \\
\hline Res. 370/97 & SENASA & $\begin{array}{l}\text { Requisitos para exportar a Europa (en relación con las directivas } \\
96 \text { y } 22 \text { de la CEE). }\end{array}$ \\
\hline Res. 345/98 & SENASA & Impone el uso de un formulario determinado para exportar a la UE. \\
\hline Res. $439 / 98$ & SENASA & Rótulos por anabólicos prohibidos para la UE. \\
\hline Res. 1912/00 & SENASA & $\begin{array}{l}\text { Exige una declaración jurada del productor que avale que los } \\
\text { bovinos para exportación a la UE no fueron tratados con } \\
\text { hormonas. }\end{array}$ \\
\hline Res. 178/01 & SENASA & $\begin{array}{l}\text { Establece la reglamentación de procesos que garanticen la } \\
\text { identificación del origen de los animales susceptibles a la fiebre } \\
\text { aftosa que se movilicen con cualquier destino. Esta norma afecta a } \\
\text { todo el ganado bovino, ya sea con destino al mercado nacional o } \\
\text { internacional. }\end{array}$ \\
\hline Res. 496/01 & SENASA & $\begin{array}{l}\text { Determina normas a las que se deben ajustar los titulares de } \\
\text { explotaciones para inscripción de establecimientos rurales } \\
\text { proveedores de ganado para faena con destino a la UE. }\end{array}$ \\
\hline Res. 155/02 & SENASA & $\begin{array}{l}\text { Crea el registro de profesionales veterinarios habilitados para el } \\
\text { despacho de faena con destino a la UE. }\end{array}$ \\
\hline Res. 15/03 & SENASA & $\begin{array}{l}\text { Se crea el "Sistema de identificación de ganado bovino para } \\
\text { exportación obligatorio". Esta reglamentación no solo exige la } \\
\text { identificación del ganado, sino que establece exigencias de } \\
\text { seguimiento. }\end{array}$ \\
\hline Res. 391/03 & SENASA & $\begin{array}{l}\text { Establece la diferencia entre establecimientos de origen y } \\
\text { establecimientos proveedores de ganado para faena (de engorde) } \\
\text { para asegurar la trazabilidad con fines sanitarios a la UE y } \\
\text { determina la trazabilidad obligatoria para todo establecimiento de } \\
\text { cría para exportación. Si bien esta norma solo se ocupa de la } \\
\text { trazabilidad para la exportación a la UE, es la primera en } \\
\text { establecer un sistema de trazabilidad integral en la Argentina. }\end{array}$ \\
\hline Res.310/04 & SENASA & $\begin{array}{l}\text { Enuncia los requisitos que deben cumplir los establecimientos } \\
\text { para faena y/o proceso y/o depósito interesados en exportar } \\
\text { carnes frescas y/o menudencias. }\end{array}$ \\
\hline Res. 754/06 & SENASA & $\begin{array}{l}\text { Establece la obligatoriedad de identificación de todo ternero en la } \\
\text { Argentina. Se crea el CUIG (clave única de identificación } \\
\text { ganadera). }\end{array}$ \\
\hline Colectiva 15/07 & SENASA & $\begin{array}{l}\text { Extiende la posibilidad de identificar dentro del mismo sistema a } \\
\text { todo el ganado bovino, pero de manera voluntaria. }\end{array}$ \\
\hline Res. 55/12 & SENASA & $\begin{array}{l}\text { Crea un subrregistro específico en el marco del Registro de } \\
\text { Establecimientos Pecuarios para engorde a corral proveedores de } \\
\text { bovinos para faena con destino a exportación. Las explotaciones } \\
\text { ganaderas que produzcan ganado para faena de este contingente } \\
\text { deberán inscribirse en este subrregistro, antes de recibir el } \\
\text { ganado. }\end{array}$ \\
\hline
\end{tabular}

Fuente: elaboración propia. 


\section{Cuota 481}

A partir de la Comunicación 2014/C N. ${ }^{\circ} 336 / 02$ de la Comisión Europea, la República Argentina ha quedado autorizada para expedir certificados de autenticidad en el marco del biotipo establecido por el Reglamento de Ejecución UE N. ${ }^{\circ} 481 / 2012$, referente a la exportación de carnes deshuesadas "High Quality Beef" procedentes de establecimientos de engorde a corral (feed lot) con destino a la Unión Europea.

El cupo anual es de 48.200 toneladas (año agrícola) repartidas trimestralmente en subcuotas de 12.050 toneladas de peso producto, entre todos los países habilitados por la citada Comisión bajo el criterio administrativo "Primero llegado, primero servido". Dicha cuota se encuentra libre de aranceles. A la fecha, además de la Argentina, se encuentran habilitados dentro de dicho contingente Australia, Canadá, Nueva Zelanda, Estados Unidos y Uruguay.

Para acceder a dicho cupo tarifario, el solicitante deberá cumplir con los requerimientos de la Resolución Conjunta N..$^{\circ} 466$ y N..$^{\circ} 361$ del Ministerio de Agricultura, Ganadería y Pesca, y del Ministerio de Economía y Finanzas Públicas, respectivamente. Asimismo, deberá cumplimentar los procedimientos establecidos por la Resolución N. ${ }^{\circ}$ 145/2014 del Ministerio de Agricultura, Ganadería y Pesca.

\section{Inscripción de la hacienda: marcas y señales}

En primer lugar, lo que debe hacer un productor ganadero para identificar su hacienda es inscribir su marca. La reglamentación acerca de este procedimiento corresponde a cada provincia. Es obligatorio para todo propietario marcar su ganado mayor y señalar su ganado menor (Ley Nacional de Marcas y Señales, 22939/83). El incumplimiento, privará al propietario de los animales de los derechos que la Ley 22939 le acuerde, referentes al régimen de propiedad del ganado, sin perjuicio de las multas que establecieren las legislaciones locales.

La marca es un dibujo, diseño o signo impreso a hierro candente o por procedimiento que produzcan análogos efectos, siempre que estén autorizados por el organismo sanitario competente. La marca deberá tener 
una dimensión máxima de diez centímetros y mínima de siete en todos sus diámetros (art. 116/117, Ley 22939). Se concede por el término de diez años a partir de su registro, pero podrá conservarse por otros términos iguales por renovaciones sucesivas (art. 122, Ley 22939).

El derecho sobre ellas se prueba con el boleto expedido por el organismo competente, o en su defecto, por las constancias de sus registros. Este derecho se pierde por no haberse utilizado en el término de 3 años a partir de su inscripción en el organismo competente (art. 123/124, Ley 22939).

Como requisito esencial y previo a todo trámite, se requiere acreditar el carácter de propietario $\mathrm{u}$ ocupante legal de un inmueble rural en la provincia (art. 126, Ley 22939). La renovación deberá ser solicitada dentro del término de un año a partir de la fecha de su vencimiento (art. 131, Ley 22939). El ganado mayor debe ser marcado antes de cumplir el año y el ganado menor señalado antes de cumplir los seis meses de edad. Está prohibido contramarcar (art. 148, Ley 22939).

En la provincia de Buenos Aires, el productor debe registrar a su nombre las marcas o señales y solo pueden ser usadas por quien las registre (art. 112/113, Código Rural de la Provincia de Buenos Aires, CRPBA, Ley 10081/83). La registración debe hacerse en el Ministerio de Asuntos Agrarios de la Provincia de Buenos Aires, en el Departamento de Registro Ganadero, que es el organismo competente y único responsable de los sistemas de diseño y de la reglamentación del uso de las marcas y señales de ganado (art. 115, CRPBA).

Todo acto jurídico mediante el cual se transfiere la propiedad de ganado mayor deberá instrumentarse con un certificado de adquisición que, otorgado por las partes, será autenticado por la autoridad local competente.

\section{Registro Nacional Sanitario de Productores Agropecuarios (RENSPA)}

Con el objetivo de regular el mercado de carnes desde el punto de vista sanitario, comercial y fiscal, el Estado Nacional creó en 1997 el Registro Nacional Sanitario de Productores Agropecuarios (RENSPA). Todos los productores pecuarios deberán inscribirse en el RENSPA (disposición conjunta 13/2007 de la Dirección Nacional de Protección Vegetal del 
SENASA y la Dirección Nacional de Fiscalización Agroalimentaria del organismo). A través de un código, se asocia a un productor con el campo donde realiza su actividad. El mencionado registro releva los datos del establecimiento y del productor: qué tipo de actividad desarrolla, así como las altas y bajas de animales del establecimiento. También realiza un control de las plagas de la ganadería, el control de las acciones sanitarias, vacunaciones, identificación, muestreos y controles fronterizos.

Si en un mismo establecimiento coexisten varios productores, cada uno deberá tener su número de identificación RENSPA. Si, en cambio, un mismo productor produce en varios establecimientos, tendrá un RENSPA por cada unidad de explotación.

\section{Clave Única de Identificación Ganadera (CUIG)}

El sistema de identificación bovina fue creado por Res. 103 de la SAGPyA (Secretaría de Agricultura, Ganadería, Pesca y Alimentación) en marzo de 2006 y reglamentado por el SENASA mediante la Res.754/2006. A partir del $1 / 1 / 2007$, todos los terneros y terneras deben estar identificados como requisito indispensable para cualquier traslado. La CUIG es usada para la identificación individual de los animales en la caravana correspondiente. La responsabilidad de obtenerla es del productor, ya sea al destete o en oportunidad de mover sus terneros, lo que antes ocurra. La CUIG establece un número abreviado del RENSPA. Al mismo tiempo, forma parte del número individual del ternero y asocia al establecimiento y al productor con el animal al momento de su nacimiento, destete o primer movimiento.

Posteriormente, SENASA, a través de la Res. 563/12, obliga a los productores a identificar a todos los animales pertenecientes a las categorías mayores de las especies bovinas, nacidos antes de la promulgación de la Resolución 754/06.

\section{Documento de Traslado Electrónico (DTE)}

Este documento reemplazó al DTA, el cual a su vez había reemplazado al antiguo Permiso Sanitario para el tránsito de los animales. Tiene como fin 
impedir el traslado de los animales de aquellos productores que no han cumplido con las pautas de vacunación. Se emite al momento de trasladar a los animales para su venta o hacia otro establecimiento agropecuario para continuar con su proceso productivo. Una vez recibida la hacienda en el establecimiento, el productor tiene la obligación de informar a la oficina del SENASA la recepción de los animales en el término de 48 horas, procediendo a invalidarse el DTE correspondiente.

\section{Guía única de Traslado ganadero}

Acompañando al DTE, en la provincia de Buenos Aires, se debe emitir la Guía única de Traslado, trámite que se realiza en la Municipalidad del partido al cual pertenece el establecimiento de origen de los animales. La norma que reglamenta este procedimiento es la Ley Provincial 10891/90. Establece que el transportista, luego de cargar la hacienda, colocará los precintos en las puertas del camión y controlará que su numeración y su color sean coincidentes con los que figuran en la Guía de Traslado.

Al dorso de la Guía única de Traslado, deberá consignar los siguientes datos: a) nombre y apellido; b) número de documento de identidad; c) número de licencia de conductor; d) número de patente del camión y de la jaula; e) de utilizar acoplado, constará también su número de patente; f) a continuación, firmará la Guía dando carácter de declaración jurada a los datos consignados.

A su vez, el remitente o su representante, controlará el cumplimiento, por parte del transportista, de las prescripciones establecidas y procederá a firmar la Guía en prueba de conformidad, dando así despacho a la hacienda embarcada. A partir de ese momento, el transportista se convertirá en el principal responsable de la carga transportada. En el caso de arreo, deberá constar solamente el número de documento y el nombre y apellido del encargado de la tropa en tránsito.

Toda hacienda que ingrese a los frigoríficos o mataderos para ser faenada deberá hacerlo en camiones precintados, provengan de remates-feria, de mercados concentradores o de establecimientos ganaderos, aunque estén situados en el mismo municipio. Serán responsables directos del 
incumplimiento de esta disposición la policía destacada en la planta, como así también los propietarios, los arrendatarios o las firmas que tengan el uso o la explotación de los respectivos frigoríficos o mataderos donde ingresa la hacienda.

No podrán formarse tropas de camiones que transporten haciendas en pie con una sola Guía de Traslado. Cada transporte deberá transitar con su pertinente Guía. Por convenio con el Gobierno nacional, podrá exceptuarse de lo citado a las haciendas provenientes del Mercado de Hacienda de Liniers, en razón de la magnitud de las compras que efectúan los frigoríficos y que deben tener pronto despacho para una rápida evacuación. En lugar de una Guía para cada camión que salga del Mercado, podrán llevar la siguiente documentación: a) un remito extendido por la Oficina de Guías del Mercado donde constarán todos los datos exigidos para las guías provinciales, apartados a), b), c), d), e) y f); b) en los mismos, constará el número de Guías a la cual pertenece, como así también la cantidad y el tipo de la hacienda que transportan (novillos, vacas, vaquillonas, etc.); c) estos remitos llevarán numeración correlativa y los resguardos de seguridad dispuestos para la Guía; d) el convenio contemplará que la Dirección del Mercado disponga los medios y el personal necesario para el control de la carga y expedición, firma de los remitos y colocación de los respectivos precintos cuyos números figurarán en el remito de la carga que lleva el transportista; e) los remitos en destino se archivarán con las Guías correspondientes; f) la policía bonaerense destacada en las plantas faenadoras procederá con los remitos, en igual forma que lo especificado para las Guías y Certificados.

Para el caso de los terneros apartados de las madres, circularán con la correspondiente Guía única de Traslado, en la que deberá constar, en los lugares reservados para los dibujos de marca, la siguiente leyenda: "Son terneros orejanos cuyas madres viajan amparadas por la Guía número... de la Municipalidad de...".

En caso de que por razones de fuerza mayor el transportista tuviera que romper el precinto original de su camión (animales muertos, caídos, rotura de camión, etc.), en el puesto policial más próximo (Caminera o Comisaría), deberá denunciar el hecho, y la Policía, previo control de la hacienda 
transportada, procederá a reponer los precintos rotos, cuya numeración será consignada en la Guía, firmada y sellada por el agente interviniente. Los precintos originales y supletorios serán archivados en destino junto con la Guía de Campaña. El suministro de precintos lo hará la provincia a los municipios, y estos a la policía de su distrito, previa toma de numeración.

El personal policial destacado para el control en destino de la hacienda, ya sea en frigoríficos, mataderos, remates-feria o mercados concentradores, procederá a anular la Guía mediante la colocación que dirá: "traslado cumplido"; le pondrán lugar y la fecha de recepción y la firmará; en los movimientos de hacienda de invernada, esta operatoria la efectuará la Oficina de Guías al archivar las mismas.

Cuando las Guías de Traslado amparen animales que se transporten con destino a invernar, el destinatario, al cumplimentar con el archivo de la Guía, entregará también los precintos del camión para su control y archivo municipal. Igual procedimiento deberán efectuar las casas de remates-feria y consignatarios al entregar la documentación a las respectivas municipalidades. En todos los casos, se cumplirá con lo referenciado en el párrafo anterior.

Los establecimientos faenadores de animales, las casas de remates-feria y los mercados concentradores de hacienda deberán arbitrar los medios necesarios para el efectivo control de la hacienda ingresada, como así también prestar la más amplia colaboración para las inspecciones de los organismos de fiscalización. La Policía destacada en establecimientos faenadores dejará expresa constancia en las Guías de Traslado cuando faltaren animales para completar el número en ellas estipulado. En estos establecimientos, en caso de inspección, deberá coincidir el número de cabezas faenadas con la cantidad policial certificada al ingreso de la planta. Igual procedimiento se seguirá con los certificados de venta.

Cuando la hacienda provenga de otra provincia, en el primer puesto policial el transportista hará controlar la carga. Para ello, la autoridad policial procederá a precintar las puertas de carga y descarga del transporte, dejándose constancia de los precintos colocados y su numeración, consignándose también al dorso de la Guía todos los datos personales y del transporte. 
Cuando la hacienda provenga de remates-feria, o de consignatarios de mercados concentradores del ámbito de la provincia de Buenos Aires donde en las respectivas Guías figuren los mismos como remitentes, no será necesario poner los números correspondientes a certificados de remisión de marcación, de boletos de marca, de certificados de compra, etc., en razón de que estas documentaciones han sido requeridas para la entrada a ferias o mercados concentradores. En estos casos, las Guías deberán llevar el sello de la casa consignataria o de remates-feria y el sello aclaratorio de la persona autorizada a firmar esta documentación.

La autoridad de aplicación de la ley es el Ministerio de Asuntos Agrarios y Pesca de la Provincia de Buenos Aires. El incumplimiento de lo estipulado en la ley por parte de los transportistas, propietarios y arrendatarios, y por los que poseen el uso o explotación de las plantas frigoríficas y de mataderos por transportar los primeros y permitir el ingreso los segundos de hacienda cuya documentación no reúna los requisitos establecidos en la ley, dará lugar a la aplicación de multas, previa instrucción del sumario correspondiente. En los casos que se detecten anomalías que supongan hacienda de dudosa procedencia, se dará intervención a la autoridad de aplicación y a la Policía. El Ministerio de Asuntos Agrarios y Pesca y el Ministerio de Gobierno implementarán las medidas necesarias para dar estricto cumplimiento a los controles que se efectúen en el tránsito de la hacienda, en especial en los establecimientos faenadores.

\section{Sistema Fiscal de Trazabilidad Animal (SIFTA)}

A través de la RG 3649 de fecha 11/7/2014 la AFIP (Administración Federal de Ingresos Públicos) establece el Sistema de Trazabilidad Animal (SIFTA). En sus considerandos, el organismo expone que a los efectos de optimizar los controles fiscales de la actividad ganadera bovina, resulta necesaria la creación de un registro que permita conocer la trazabilidad de la hacienda. La citada resolución, en su art. 9..$^{\circ}$ reglamenta que el sistema entrará en vigencia a partir del 1/1/2015, siempre y cuando el organismo, tal como lo enuncia en su art. $8 .^{\circ}$, defina todos aquellos aspectos complementarios y necesarios para la operatividad del sistema que se implementa por dicha 
resolución, entre los cuales menciona los alcances funcionales y tecnológicos, los plazos y vigencias, las características del dispositivo a utilizar, los sujetos obligados y la información a suministrar. Dado que a la fecha de redacción del presente trabajo, la AFIP aún no definió los aspectos que se acaban de mencionar, esta norma no ha entrado en vigencia.

En opinión doctrinaria de Pampliega (2014) es necesario hacer hincapié en dos cuestiones relevantes. En primer lugar, se debe considerar el quiebre del equilibrio entre el sistema de autodeterminación que rige la materia tributaria y la proliferación sin aparente límite de los regímenes de información que no solo generan costos, sino que modifican el objeto comercial de los actores del sistema, desplazando el objeto primario en derivaciones que deberían cumplir un principio básico del derecho tributario, cual es el de neutralidad.

Por otro lado, debe tenerse presente que si bien las facultades reglamentarias se reservan en cabeza de la AFIP, se estima que razonabilidad y legalidad mediante, los datos que integren el referido régimen estarán comprendidos dentro del instituto del secreto fiscal que prevé el art. 101 de la Ley 11683 (TO en 1998 sus modificaciones y complementarias).

Habiendo aclarado estos puntos, Pampliega (2014) considera que el sistema se enmarca en la creciente implementación de tecnología para reducir la brecha de la evasión fiscal, que en este nicho es conocida por todos los profesionales y actores económicos como cuasi endémica. A su vez, el autor plantea que podría redundar a futuro en una trazabilidad que trascienda las fronteras de las conductas fiscales, pudiendo identificar lugares de pastoreo y zonas eventualmente con certificación libre de aftosa, con el impacto trasnacional que ello implicaría para las exportaciones argentinas en mercados con esos requisitos. El autor señala que sería interesante evaluar si no se puede llegar al mismo resultado sin la implementación de este régimen, valiéndose de información ya existente en el circuito y que dispone el SENASA sobre vacunación, en tanto tiene injerencia directa en el frigorífico que faena. 


\subsubsection{Situación normativa en Brasil y Uruguay}

Para evaluar la situación de la Argentina en el contexto internacional de las regulaciones en materia de trazabilidad bovina, se efectúa un análisis de las principales presiones coercitivas vigentes en los dos países exportadores de carne históricamente más importantes de América del Sur: Brasil y Uruguay (MINAGRI, 2015). Cabe aclarar que no es intención del presente trabajo efectuar un estudio comparado de las distintas presiones institucionales con dichos países, sino conocer el grado de avance en aquellas demandas coercitivas en las que el Estado tiene injerencia, dado que pueden condicionar la implementación eficaz del sistema, a pesar de las normas o prácticas voluntarias que otros actores del campo organizacional desarrollen (presiones normativas o miméticas).

En Brasil, el sistema de trazabilidad bovina utilizado se denomina SISBOV (Sistema Brasileiro de Identificação e Certificação de Bovinos e Bubalinos) y tiene vigencia desde el año 2002. Consiste en certificar una serie de acciones para garantizar la sanidad y seguridad del alimento proveniente del ganado brasilero. A partir de este sistema, es posible efectuar el seguimiento del producto dentro de toda la cadena de la carne, desde el campo hasta el frigorífico, garantizando la calidad del producto para el consumidor/comprador.

Toda la cadena de la carne está involucrada en la ejecución del sistema. Participan productores rurales, organizaciones certificadoras, fábricas de elementos de identificación, frigoríficos, oficinas locales de atención veterinaria y el Ministerio de Agricultura, Ganadería y Abastecimiento (MAPA). El productor no está obligado a adherir al SISBOV. La obligatoriedad existe cuando se comercializa con mercados que exigen la trazabilidad.

Para adherir al sistema, los productores necesitan que se registre tanto el productor como el establecimiento donde se efectúa la producción. Además debe:

- Presentar un protocolo básico de producción.

- Registrar los insumos utilizados para producir.

- Identificar individualmente el $100 \%$ del ganado. 
- Efectuar un seguimiento del movimiento de los animales.

- Estar supervisado por una certificadora inscripta dentro del MAPA para tal fin.

- Recibir visitas periódicas de la citada certificadora. Si no se realizan las visitas periódicas, se pierde la certificación del establecimiento.

El plazo mínimo de permanencia de un animal en zona habilitada, a los efectos de ser clasificado como apto para mercados que exigen trazabilidad, es de 90 días y el animal debe permanecer en un establecimiento aprobado por el SISBOV, por lo menos, los últimos 40 días antes de la faena (SERPRO, 2008).

Quien tiene su propiedad inscripta en el SISBOV, cuando vende sus animales para faena, obtiene un precio superior, cercano al $10 \%$ de incremento, en relación con aquellos animales que no están trazados. La realidad actual del mercado brasilero es que, aun con el beneficio descripto, la adhesión de los productores al SISBOV está prácticamente paralizada (Mota, 2011).

Se han identificado los siguientes factores que afectan la adhesión voluntaria al SISMOV: costos altos de trazabilidad para los productores; inestabilidad del sistema a través de circulares que son publicadas constantemente, anulando las anteriores y alterando los procedimientos y exigencias por parte del MAPA; en virtud de las razones anteriores, dificultades de interpretación de las normas por las organizaciones certificadoras y frigoríficos; falta de confiabilidad del sistema de rastreo a través de etiquetas o caravanas; e insuficiente entrenamiento y capacitaciones para su correcta implementación a lo largo de la cadena (Curti Rodrigues \& Diniz Nantes, 2010).

En lo que respecta a Uruguay, este país implementó a partir del año 2006 un Sistema de Identificación y Registro Animal (SIRA), a través de la Ley 17997, luego modificada por la 18656 de abril del año 2010 y la Resolución 35/2011 de marzo del año 2011.

Comenzó como un plan sanitario piloto que acompañaría el nuevo acuerdo comercial con Europa para comercializar cuota Hilton. En un inicio, se identificaban los animales en forma grupal, pasando paulatinamente, y luego de mucho trabajo, a la identificación individual que requería Europa. 
A partir del $10^{\circ}$ de abril de 2010, en la cuota Hilton, solo se aceptaron animales trazados individualmente y desde el 1..$^{\circ}$ de julio de 2011 se generalizó la trazabilidad a toda la hacienda uruguaya, sancionando a los productores con la faena inmediata de aquellos animales que no estuvieran incorporados al sistema.

Hoy Uruguay posee un sistema de trazabilidad obligatorio para todos los productores ganaderos del país. El detentar este estatus de seguimiento sanitario ha colocado a Uruguay en un lugar de gran prestigio en el mundo respecto de las garantías que ofrece como país exportador de alimentos (Toro, 2009).

\subsection{Presiones normativas y miméticas sobre trazabilidad en la cadena bovina}

La trazabilidad puede ser concebida como una tecnología en el sentido de una práctica social destinada a modificar el mundo físico o social según rutinas o métodos identificables (Bonet de Viola, 2012). Esas prácticas pueden estar receptadas en la regulación normativa o bien ser voluntariamente aplicadas en la realidad operativa del negocio.

Al respecto se observa que, de las disposiciones vigentes, surge la existencia de un doble estándar sanitario y comercial, por el cual son mayores las exigencias requeridas para abastecer el mercado de exportación respecto del consumo interno. Las carnes para mercado interno no tienen obligación de seguimiento, aunque sí se cumple la identificación de terneros. No obstante lo dispuesto por la regulación, la trazabilidad puede emplearse como un componente de los sistemas de certificación de calidad y un instrumento para la gestión de las cadenas de aprovisionamiento (Supply Chain Management).

Palau (2006), en un estudio realizado de los sistemas de aseguramiento de origen y calidad en las carnes vacunas argentinas, encontró que a nivel institucional los temas de la trazabilidad y de los protocolos de origen están en una etapa de iniciación. Tampoco hay en el país un gran número de asociaciones de productores aplicando estos sistemas. Un caso que se ha mantenido en el tiempo es el de la marca colectiva "Argentine Angus Beef", a 
través del esfuerzo de la Asociación Argentina de Angus. Durante el año 2013 se comercializaron $4.377 \mathrm{t}$ de carne certificada para el mercado interno y externo con destino a Alemania, España, Italia, Holanda, Brasil, Dubai y Chile, aumentando en aproximadamente un $30 \%$ el volumen comercializado en relación con el ejercicio anterior, logrado principalmente por el aumento de la comercialización en los fastfood (Asociación Argentina de Angus, 2013).

Los principales motivos de la escasa adopción de estos programas en la Argentina y su baja participación en el mercado interno y externo son el exiguo cumplimiento de los contratos y de la ley, la escasa capacidad de los productores y agentes comerciales de formar redes debido a ello, la baja participación de las exportaciones de carne y la no disposición de la gran mayoría de la población a pagar sobreprecios por carnes certificadas, aun cuando existen algunos nichos (Palau, 2006).

En orden a lo analizado por este autor, Champredonde, Vitrolles, Casabianca \& Cerdan (2014) refuerzan la influencia que tienen las regulaciones en materia de trazabilidad como base para la aplicación de programas de carnes certificadas. Exponen el caso de proyecto "Carnes Pampa del Salado", resaltando que una primera amenaza externa fue la implementación del sistema oficial de trazabilidad impuesto por SENASA, destinado inicialmente a identificar únicamente el ganado de exportación. Si bien se asemejó al sistema desarrollado por el Consorcio, el sistema oficial no exigió al resto de los productores pampeanos el mismo nivel de generación y comunicación de información (relativa al proceso de producción) que se autoimponían los productores involucrados en este proyecto. La implementación de esas medidas oficiales llevó a los actores implicados a interrogarse sobre el futuro del Consorcio, dado que los compradores no asignaban valores diferenciales a los productos trazados y con información, y fue una de las causas determinantes del retiro de sus participantes.

El autor coincide con Palau (2006) en que aún existen problemas de estabilidad y claridad en las reglas de juego para operar en el mercado externo e interno, que no contribuyen a incentivar el interés de los participantes de la cadena por programas de calidad certificada. En el mismo sentido, Garrido (2006) analiza que un punto vital que debería cumplir el sistema de trazabilidad 
argentino es la comunicación, mediante una adecuada captura de datos que pueda conformar una base nacional y sea el engranaje de los distintos eslabones que actúan en la cadena de valor de la carne. Sin embargo, para invertir en tecnología que la sustente, considera necesario que el Estado asuma un papel proactivo en los proyectos de trazabilidad, relevando experiencias privadas, promoviendo los mercados y estableciendo medidas que faciliten el seguimiento del animal hasta el consumidor, condiciones que aún no se han logrado efectivamente.

Sobre la base de los estudios anteriores puede inferirse que las presiones normativas en favor de un sistema trazado son débiles. A través de entrevistas a referentes calificados del campo organizacional de la cadena de la carne bovina, se pudieron confirmar estas hipótesis y valorar la interpretación cognitiva que esos actores realizan de la trazabilidad y su puesta en práctica en la región de estudio, que tiene amplia tradición en ganadería, actividad destacada en la Ley del SO bonaerense.

En primer término, se indagó si la trazabilidad según las regulaciones vigentes se encuentra desarrollada en la Argentina como un sistema completo que pueda garantizar el origen y la calidad de la carne en todas las etapas de su comercialización. El productor ganadero manifestó la visión más pesimista, al considerar que

representa una carga administrativa que implica un costo, porque la exportación no funciona y en el mercado interno no se aplica. Existen problemas operativos de conciliación entre los registros del productor y de SENASA. Además, se percibe un interés fiscal más que sanitario, para tener actualizado el número de cabezas existentes en los establecimientos.

Por su parte, tanto el consignatario como el funcionario de SENASA coincidieron en afirmar que el sentido de la trazabilidad es sanitario, y secundariamente puede ser fiscal. Expusieron que la caravana no resulta un dispositivo apropiado de identificación animal (se cae, tiene problemas de lectura, etc.), lo cual torna el sistema engorroso, generando un costo de tipo administrativo, más que económico (dado que el valor de la caravana es bajo). Asimismo, el productor muchas veces no cumple con las obligaciones 
de identificación y registro con el debido cuidado. Ello sumado a que no hay exportación, no genera incentivos para mantener los animales trazados.

Sin embargo, el consignatario manifestó que desde ese canal comercial se estimula la trazabilidad. Expresó:

El 80\% de los criadores que venden terneros por su intermedio están trazados. Aun cuando no hay exportación, el productor de carne de exportación para faena continúa trazando por si se abre el mercado externo. Dado que si no opera por dos años con ese destino, se cae la certificación del campo y hay que comenzar de nuevo. Por lo tanto, busca comprar animales trazados y procura vender alguna jaula para la exportación para evitar la pérdida del estatus exportador.

El funcionario del SENASA indicó que por Res. 15/03 deben inscribirse los establecimientos proveedores de carne para la exportación, y a través de la Res. 391/03 se obliga a los criadores de animales con ese destino a identificar a todos sus terneros. Solo se obliga a los de esta categoría, no incluyendo otras del rodeo reproductor. Aquí nace un doble estándar. Si un criador se inscribe de acuerdo a la Res. 391/03 y mueve sus terneros para engordar fuera de su establecimiento de origen hacia otro, que también es de su propiedad o arrienda, se pierde la trazabilidad, y por ende la posibilidad de vender estos animales a un productor inscripto de acuerdo a la Res. 15/03. Por lo tanto, solo puede comercializar sus animales en el mercado interno. El entrevistado postuló que la Res. 391/03 debería ser más flexible y contemplar esta realidad, de manera de unificar todo el sistema de trazabilidad y no generar distintas categorías de animales, por el solo hecho de trasladarlos para proseguir su engorde a otro establecimiento, hecho que ocurre muy a menudo en la Argentina.

El representante del frigorífico integrado a un supermercado regional también expresó que

la trazabilidad tiene lógica en la exportación, porque hay un mercado externo que lo exige. En el mercado interno no se aplica. La trazabilidad se corta en el momento de la faena. Al frigorífico que abastece el mercado interno no le es determinante en la compra de 
hacienda que los animales sean trazados. La calidad se busca a través de otros medios, efectuando un scoring propio mediante evaluación de proveedores según la homogeneidad de su oferta y confiabilidad. El consumidor argentino aún no paga por trazabilidad, incluso el diferencial de precio a obtener por una marca en el mercado interno masivo (no de nichos) no es significativo, puesto que la calidad intrínseca de la carne es alta y el consumidor puede valorarla solo por tradición, sin necesidad de otros signos. Esto a pesar de los estándares bajos que existen para el mercado interno, y las diferentes habilitaciones sanitarias de tránsito (federal, provincial o municipal) que no favorecen la formalidad y la lealtad comercial.

Finalmente, todos los entrevistados consideraron que la certificación de calidad de las carnes tiene futuro, dado que puede generar beneficios comerciales para un mejor posicionamiento en el mercado externo e interno dentro de ciertos nichos. Incluso se trata de animales que se terminan mejor y más rápidamente, pagando el invernador un diferencial de precio. Con la trazabilidad se garantiza el origen. Según el representante del frigorífico:

La trazabilidad asegurará calidad en la medida que se adopten medidas correctivas tendientes a la mejora continua. Además la complejidad que implica la administración de la información, requerirá un software adecuado que emplee un mismo lenguaje en toda la cadena de valor, condición que actualmente no se da. Por otra parte, no debieran en ese contexto existir controles de precios para algunos cortes finales, puesto que no favorecen las estrategias de diferenciación al comercializarse medias reses.

A continuación, se presenta en la tabla 2 un resumen de los resultados de las entrevistas realizadas: 
Mauricia Nori, Liliana Scoponi, Regina Durán, Marcelo Fernandes Pacheco Dias

Tabla 2. Resultados de las entrevistas a actores de la cadena bovina del SO bonaerense

\begin{tabular}{ccccc}
\hline Temas & $\begin{array}{c}\text { Productor } \\
\text { ciclo } \\
\text { completo }\end{array}$ & $\begin{array}{c}\text { Consignatario } \\
\text { de hacienda }\end{array}$ & $\begin{array}{c}\text { Frigorífico } \\
\text { integrado a } \\
\text { supermercado }\end{array}$ & $\begin{array}{c}\text { Funcionario } \\
\text { SENASA }\end{array}$ \\
\hline $\begin{array}{c}\text { Trazabilidad: ¿de acuerdo a las } \\
\text { disposiciones vigentes, funciona como } \\
\text { sistema completo para el } \\
\text { aseguramiento de la calidad? }\end{array}$ & No & $\begin{array}{c}\text { Sí, para } \\
\text { mercado } \\
\text { externo }\end{array}$ & No & No \\
\hline $\begin{array}{c}\text { Trazabilidad: ¿costo u oportunidad de } \\
\text { mejores precios? }\end{array}$ & Costo & Ambos & Costo & Ambos \\
\hline $\begin{array}{c}\text { Programas de calidad de carnes } \\
\text { certificadas: ¿generan beneficios? }\end{array}$ & $\begin{array}{c}\text { No generan } \\
\text { beneficio } \\
\text { actual, pero } \\
\text { tienen futuro. }\end{array}$ & $\begin{array}{c}\text { Generan } \\
\text { beneficios }\end{array}$ & $\begin{array}{c}\text { Generan } \\
\text { beneficios }\end{array}$ & $\begin{array}{c}\text { Generan } \\
\text { beneficios }\end{array}$ \\
\hdashline $\begin{array}{c}\text { Normativa actual sobre identificación } \\
\text { y trazabilidad: iqué aspectos } \\
\text { privilegia? }\end{array}$ & Fiscal & $\begin{array}{c}\text { Sanitario y } \\
\text { Fiscal }\end{array}$ & Sanitario y \\
Fiscal & Sanitario y \\
\hline
\end{tabular}

Fuente: elaboración propia.

\section{Conclusiones}

Como consecuencia de la necesidad de adecuarse a la normativa europea sobre trazabilidad, la Argentina ha ido generando regulaciones, al igual que Brasil y Uruguay. De manera paulatina surgió en el país una profusa normativa que torna el sistema complejo y no le confiere un carácter holístico, en el cual se mantiene un doble estándar con menores exigencias para el mercado interno respecto del externo.

En la gestión organizacional, la trazabilidad es percibida por los agentes de la cadena del SO bonaerense como una carga administrativa, la cual surge de los problemas operativos que plantea el uso de la caravana, a pesar de su bajo costo, ya que no es un dispositivo confiable. Y por otra parte, tiene su origen en la gran cantidad de normas a cumplir en diferentes jurisdicciones (municipal, provincial, nacional). Similares dificultades se advierten en Brasil; no así en Uruguay, que ha generado un sistema coercitivo exitoso que crea condiciones para el desarrollo de programas de carne certificada.

Por otra parte, el doble estándar sanitario no contribuye a la formalidad y la competencia en condiciones de igualdad dentro de la cadena. Esta situación, sumada al cierre acontecido en varias oportunidades de las exportaciones y la incertidumbre sobre el futuro de las políticas de comercio 
exterior, desalienta a los productores registrados a permanecer trazados y amenaza la actividad de los frigoríficos.

Se encontró coincidencia en la consideración de que el mercado interno no valora en general los beneficios de la trazabilidad a través del pago de un precio superior. La calidad de la carne que se consume es alta a pesar del bajo estándar interno. Además, por cultura y tradición, el consumidor masivo conoce el producto y no demuestra interés por verificar la calidad de origen. Tampoco favorece la adopción, aún voluntaria, de la trazabilidad para la gestión de calidad certificada, que se fijen disposiciones de control de precios en algunos cortes, en virtud de la comercialización de medias reses.

Así, son varios los factores que inciden para que las presiones normativas y cognitivas sean débiles en favor de la aplicación de la trazabilidad para el aseguramiento de la calidad de la carne. En síntesis, la Argentina ha avanzado en la identificación animal para control sanitario, fiscal y de propiedad de la hacienda. No obstante, la identificación solo representa un paso en un sistema integral de trazabilidad. El seguimiento animal y la administración de la información se cumplen en respuesta a la exigencia del mercado externo, principalmente de la UE. Los actores perciben complejas las presiones coercitivas vigentes, puesto que no hay uniformidad en las disposiciones, las cuales difieren según el destino que tenga la hacienda y la finalidad perseguida por la norma.

La presente investigación tiene las limitaciones de un estudio cualitativo que no posibilita generalizaciones de las conclusiones formuladas, por lo cual se podrían emplear otras técnicas confirmatorias en futuros estudios. No obstante, se ha procurado avanzar en la comprensión preliminar de la situación actual de la trazabilidad bovina en la Argentina y la región de estudio ante la carencia de investigaciones aplicadas de la disciplina administrativa en esta temática.

Se advierten oportunidades dada la calidad intrínseca que presenta la carne argentina, de perfeccionar y ampliar la aplicación del actual programa de trazabilidad para su empleo en sistemas de aseguramiento de la calidad, siguiendo la tendencia de los mercados mundiales. Ello supone repensar políticas públicas de largo plazo que fomenten la exportación y la expansión de los mercados, ordenen la normativa, simplifiquen aspectos 
administrativos inherentes al registro y la identificación de la hacienda, organicen sistemas de información adecuados que integren dichos registros en todos los eslabones de la cadena y consideren la equidad en la regulación de requisitos sanitarios y fiscales a cumplir por los agentes participantes del agronegocio para evitar asimetrías.

Futuras investigaciones podrán abordar la capacidad de agencia de las organizaciones involucradas en la cadena bovina regional para dar respuesta a las presiones sobre trazabilidad (Oliver, 1991; Pache \& Santos, 2010), en orden a proponer cambios que posibiliten una aplicación holística como base de sistemas de aseguramiento de la calidad. En lo que se refiere a los aspectos de la gestión de estos sistemas, a partir de los resultados hallados, se considera que el diseño de protocolos de certificación de calidad claros en materia de obligaciones y beneficios de las partes, que sea supervisados por una organización que las represente, como es el caso de la Asociación Argentina de Angus, favorecería el cumplimiento de los compromisos asumidos y la institucionalización de esas prácticas. No obstante, esta condición sería insuficiente en caso de que no se desarrollaran los aspectos institucionales antes citados relativos al funcionamiento de los mercados y el ordenamiento de las disposiciones sobre trazabilidad con visión comercial.

Asimismo, sería de interés profundizar el estudio de las demandas institucionales en Brasil y Uruguay, a través de una investigación comparada, que persiga conocer las presiones normativas y miméticas presentes en los campos organizacionales de sus cadenas de valor ganaderas, en relación con las regulaciones que esos países han desarrollado.

\section{Referencias bibliográficas}

Alburquerque, A. (2002). Teoría de la Organización y Nuevo Institucionalismo en el Análisis Organizacional. Administración y Organizaciones, 8(4), pp. 13-44. Recuperado el 20/04/2015 de http:/ / bidi.xoc.uam.mx/resumen_articulo.php?id=2764\&archivo=9-2212764mgi.pdf\&titulo_articulo=Teor\%EDa $\% 20 \mathrm{de} \% 201$ as $\% 20$

Organizaciones $\% 20 \mathrm{y} \% 20 \mathrm{Nuevo} \% 20 \mathrm{Institucionalismo} \% 20 \mathrm{en} \% 20 \mathrm{el} \% 20 \mathrm{An}$ \%E1lisis\%20Organizacional 
Aráoz, L. F. (2004). Trazabilidad de la carne bovina en la Argentina. Proyecto FAO. Países del MERCOSUR ampliado, "Apoyo a la Integración del Sector Agropecuario del Cono Sur para Contribuir a las Políticas de Seguridad Alimentaria" (TCP/RLA/2910). Buenos Aires: FAO. Recuperado el 20/04/2015 de http://www.ipcva.com.ar/files/ trazabilidad_ra.pdf

Asociación Argentina de Angus (2013). Angus nuevamente líder entre las razas bovinas. Boletín 2013. Buenos Aires: Asociación Argentina de Angus.

Bardin, L. (1977). Análise de conteúdo. Lisboa: Edições 70.

Bonet de Viola, A. M. (2012, noviembre). Sistema Normativo Argentino de Trazabilidad Bovina. En 9. Encuentro de Colegios de Abogados sobre temas de Derecho Agrario, realizadas en el Colegio de Abogados de Rosario, Rosario.

Carvalho, C. \& Vieira, M. (2003). Contribuições da perspectiva institucional para a análise das organizações: possibilidades teóricas, empíricas e de aplicação. En Carvalho, C. \& Vieira, M. (eds.), Organizações, cultura e desenvolvimento local: a agenda de pesquisa do Observatório da Realidade Organizacional (pp. 23-40). Recife: Editora UFPE.

Champredonde, M. Vitrolles, D., Casabianca, F. \& Cerdan, C. (2014). La Pampa como indicación geográfica para diferenciar carnes vacunas en Argentina y en Brasil: motivaciones y limitantes. Agroalimentaria, 20(38), pp. 35-52. Recuperado el 30/05/2015 de http://erevistas.saber.ula.ve/ index.php/agroalimentaria/article/view/5285/5075

Curti Rodrigues, L. \& Diniz Nantes, J. F. (2010). Rastreabilidade na cadeia produtiva da carne bovina: Situação atual, dificuldades e perspectivas para o Brasil. Informações Econômicas, SP, 40(6), pp. 31-41. Recuperado el 30/05/2015 de http://ftp.sp.gov.br/ftpiea/publicacoes/ie/2010/tec3-0610.pdf

De las Carreras, A. (2010). Ganados y carnes vacunas. En Reca, D., Lema, D. y Flood, C. (eds.), El crecimiento de la agricultura argentina. Medio siglo de logros y desafíos (pp. 29-65). Buenos Aires: UBA. 
DiMaggio, P. J. \& Powell, W. W. (1983). The iron cage revisited: Institutional isomorphism and collective rationality in organizational fields. American Sociological Review, 4(2), pp. 147-160. DOI: 10.2307/2095101.

Felipe Boente, I. de \& Briz Escribano, J. (2004). Seguridad y trazabilidad alimentaria en el contexto internacional. Boletín Económico de ICE 2790: pp. 41-49. Recuperado el 20/04/2015 de: http://www.revistasice.info/ cachepdf/BICE_2790.pdf

Garrido, S. A. (2006). Trazabilidad de carnes: sistemas, coyuntura y futuro. Sitio Argentino de Producción Animal. Recuperado el 20/04/2014 de http://www.produccion-animal.com.ar/produccion organica_y_trazabilidad/31-trazabilidad_comparada.pdf

Green, R. (2007). Trazabilidad de carnes en el mercado mundial. Plataforma Tecnológica Regional. Hacia el fortalecimiento competitivo de la cadena de carne bovina en la región MERCOSUR ampliado, PROCISUR-IICA, Francia, Loira: INRA. Recuperado el 20/04/2015 de http://www10.iadb.org/intal/intalcdi/pe/2010/05177.pdf

Greenwood, R. ; Oliver, C..; Sahlin, K. \& Suddaby, R. (2008). Introduction. En Greenwood, R., Oliver, C., Sahlin, K. y Suddaby, R. (Eds.). The Sage handbook of organizational institutionalism (pp. 1-46). London: Sage.

Hernández Sampieri, R., Fernández Collado, C. \& Baptista Lucio, P. (2010). Metodología de la investigación. 5. a edición. México: McGrawHill.

Heyder, M. \& Theuvsen, L. (2009, septiembre). Corporate social responsibility in agribusiness: empirical findings from Germany. En 113 th EAAE Seminar, 113, "A resilient European food industry and food chain in a challenging world", realizadas en Creta, Grecia.

Iglesias, D. \& Ghezan, G. (2010). Análisis de la Cadena de la Carne Bovina en Argentina. Estudios Socioeconómicos de los Sistemas Agroalimentarios y Agroindustriales, n. ${ }^{\circ}$ 5. Buenos Aires: INTA.

Llamas-Sánchez, R. (2005). Un análisis institucional de la implantación de la Agenda Local 21 por los ayuntamientos españoles. Tesis (Doctorado en Administración), Facultad de Ciencias Económicas y Empresariales. Universidad de Granada, España. Recuperado el 20/04/2015 de http://digibug.ugr.es/bitstream/10481/585/1/15403051.pdf 
Lopes, F. \& Baldi, M. (2013). Estratégia como contexto interfirma: uma análise a partir da inmersão social e da Teoría Institucional no setor de carcinicultura norte-rio-grandense. Administração Mackenzie (RAM), 14(2), pp. 210-242. Recuperado el 20/04/2015 de http://www.redalyc.org/pdf/ 1954/195426233009.pdf

Machado-da-Silva, C. M. \& Coser, C. (2006). Rede de Relações Interorganizacionais no Campo Organizacional de Videira-SC. Administração Contemporânea, 10(4), pp. 9-45. Recuperado el 20/04/2015 de http://www.redalyc.org/pdf/840/84010402.pdf

Meyer, J. (2008). Reflections on institutional theories of organizations. En Greenwood, R., Oliver, C., Sahlin, K. y Suddaby, R. (eds.), The Sage handbook of organizational institutionalism (pp. 790-813). London: Sage.

MINAGRI (2015). Informe del Mercado Internacional Pecuario. Dirección de Estudios del Sector Pecuario. MINAGRI, año 1, n.o 16. Buenos Aires: MINAGRI. Recuperado el 20/05/2015 de http://www.minagri.gob.ar/ site/ganaderia/bovinos/05=Mercados/

Mota, É. Gomes da (2011). A rastreabilidade bovina no Brasil: histórico, evolução $e$ perspectiva de futuro. Dissertação Mestrado em AgronegóciosUniversidade de Brasília, Brasília. Recuperado el 30/05/2015 de http:/ / repositorio.unb.br/handle/10482/8767

Oliver, C. (1991). Strategis responses to institutional processes. Academy of Management Review, 16(19), pp. 145-179. DOI: 10.2307/258610.

Pache, F. \& Santos, F. (2010). When Worlds Collide: The internal dynamics or organizational responses. Academy of Management Review, 35(3), pp. 455-476. DOI: 10.5465/AMR2010.51142368.

Palau, H. (2006). Los sistemas de aseguramiento de origen y calidad en las carnes vacunas argentinas. En Palau, H.; Ordóñez, H. \& Senesi, S. (eds.), Guía para la identificación y trazabilidad animal en las carnes argentinas. Buenos Aires: Inforcampo.

Pampliega, A. (2014). Implementación de la primera etapa del SIFTA, "Sistema Fiscal de Trazabilidad Animal" [columna de opinión, La Ley online Doctrina, Thomson Reuters, 29 de julio de 2014]. Recuperado el 20/04/2015 de thomsonreuterslatam.com/2014/07/29/columna-de- 
opinion-implementacion-de-la-primera-etapa-del-sifta-sistema-fiscal-detrazabilidad-animal-autor-pampliega-alejandro/

Ponti, D. (2011). Canales de comercialización de carne vacuna. Buenos Aires: Ministerio de Agricultura Ganadería y Pesca.

Rodríguez-Ramírez, R., González-Córdova, A., Arana, A., SánchezEscalante, A., \& Vallejo-Córdoba, B. (2010). Trazabilidad de la carne de bovino:conceptos, aspectos tecnológicos y perspectivas para México. Interciencia, 35(10), pp. 746-751. Recuperado el 31/05/2015 de http://www.redalyc.org/pdf/339/33915592006.pdf

Saunders, M., Lewis, P. \& Thornhill, A. (2011). Research Methods For Business Students, 5/e. India: Pearson Education.

Scott, W. R. (2001). Institutions and Organizations. Thousand Oak, CA: Sage. SERPRO (2008). SISBOV - Qualidade desde a Origem. Informe Temas, 195, novembro/dezembro 2008. Recuperado el 20/08/2014 de http://www4.serpro.gov.br/imprensa/publicacoes/tema-1/antigas $\%$ 20temas/tema-195/materias/sisbov/

Suddaby, R. (2010). Challenges for Institutional Theory. Journal of Management Inquiry, 19(1), pp. 14-20. DOI: 10.1177/1056492609347564.

Suddaby, R., Elsbach, K., Greenwood, R., Meyer, J. \& Zilber, T. (2010). Organizations and their institutional environments, bringing meaning, values, and culture back. En Introduccion to the special research forum. Academy of Management Journal, 53(6), pp. 1234-1240. DOI: 10.5465/ AMJ,2010.57317486.

Thornton, P. H. \& Ocasio, W. (2008). Institutional Logics. En Greenwood, R., Oliver, C., Sahlin, K. \& Suddaby, R. (eds.), The Sage handbook on organizational institutionalism (pp. 99-129). London: Sage.

Toro, G. (2009). La experiencia de Uruguay en la trazabilidad bovina. Informe IICA División de Cooperación Técnica Horizontal. Montevideo: IICA. Recuperado el 20/04/2015 de http://repiica.iica.int/docs/ B1637E/B1637E.PDF 
Trazabilidad bovina en el Sudoeste bonaerense: Reflexiones...

1 Avance PGI (Proyecto Grupo de Investigación) 24/C038 SGCyT UNS -“Gestión organizacional ante demandas institucionales en la cadena bovina del SO bonaerense" - y Proyecto 007/13 Núcleo de Estudios e Investigaciones en Educación Superior del Sector Educativo del MERCOSUR - "Vinculación universidad-empresa: análisis de las posibilidades de transferencia tecnológica para la gestión del agronegocio" - .

2 Departamento de Ciencias de la Administración. Universidad Nacional del Sur. mnori@criba.edu.ar

3 Departamento de Ciencias de la Administración. Universidad Nacional del Sur. liliana.scoponi@uns.edu.ar

4 Departamento de Ciencias de la Administración. Universidad Nacional del Sur. rduran@criba.edu.ar; regina.duran@uns.edu.ar

${ }^{5}$ Facultad de Administración y Turismo (FAT). Universidad Federal de Pelotas (UFPel), RS-Brasil.marcelo.fernandes@ufrgs.br 